\title{
Evolution of the Electron Distribution Function in the Whistler Wave Turbulence of the Solar Wind
}

\author{
V. Pierrard ${ }^{1,2}$ - M. Lazar $^{3,4}$. \\ R. Schlickeiser ${ }^{3,5}$ \\ Received ; accepted \\ (C) Springer $\bullet \bullet \bullet \bullet$
}

\begin{abstract}
The electron distribution functions from the solar corona to the solar wind (SW) are determined in this paper by considering the effects of the external forces, of Coulomb collisions and of the wave-particle resonant interactions in the plasma wave turbulence. The electrons are assumed interacting with righthanded polarized waves in the whistler regime. The acceleration of electrons in the SW seems to be mainly due to the electrostatic potential. Wave turbulence determine the electron pitch-angle diffusion and some characteristics of the velocity distribution function (VDF) such as suprathermal tails. The role of parallel whistlers can also extend to small altitudes in the SW (the acceleration region of the outer corona), where they may explain the energization and the presence of suprathermal electrons.
\end{abstract}

Keywords: Corona; Flares, Dynamics; Solar wind; Instabilities; Waves, Plasma

\section{Introduction}

The solar wind is a low density non uniform plasma in which kinetic processes prevail. Kinetic models based on the solution of the Fokker-Planck equation have been developed to study the steady state electron VDF in the corona and at larger radial distances in the SW (Lie-Svendsen et al., 1997; Pierrard et al., 1999; Lie-Svendsen and Leer, 2000; Pierrard et al., 2001; Vocks and Mann, 2003;

\footnotetext{
1 Belgian Institute for Space Aeronomy, B-1180 Brussels,

Belgium, email: viviane.pierrard@oma.be

2 Center for Space Radiations, Research Institute in

Mathematics and Physics, Université Catholique de Louvain,

Chemin du cyclotron 2, 1348 Louvain-La-Neuve, Belgium

3 Research Department - Plasmas with Complex

Interactions, Ruhr-Universität Bochum, D-44780 Bochum,

Germany, email: mlazar@tp4.rub.de

4 Center for Plasma Astrophysics, K.U.Leuven,

Celestijnenlaan 200B, 3001 Leuven, Belgium

${ }^{5}$ Institut für Theoretische Physik, Lehrstuhl IV: Weltraum-

und Astrophysik, Ruhr-Universität Bochum, D-44780

Bochum, Germany, email: rsch@tp4.rub.de
} 
Vocks et al., 2005; Vocks et al., 2008; Vocks and Mann, 2009). First, the test electrons were submitted to the influence of the external forces and to Coulomb collisions with background particles (Pierrard et al., 1999; Lie-Svendsen and Leer, 2000; Pierrard et al., 2001). Such models were solved numerically and emphasized the effects of Coulomb collisions compared to the results of purely exospheric models considering only the external forces (Maksimovic et al., 1997a; Lamy et al., 2003). They showed that Coulomb collisions have important effects on angular scattering (i.e., on the pitch angle distribution of the electrons) but do not modify their average density and mean temperatures radial distributions. Models including Coulomb collisions give more realistic VDF and a reduction of the temperature anisotropies compared to the purely exospheric approximation (Lemaire and Pierrard, 2001), because the Coulomb collisions isotropize the electron VDF. The characteristics and the acceleration mechanisms of the heavy ions have been studied by Pierrard et al. (2004).

These models also emphasize that suprathermal electrons should be present at low altitudes in the corona in order to match the observed distributions at 1 AU (Pierrard et al., 2001). The suprathermal electrons play an important role in the acceleration process of the SW. Indeed, these suprathermal electrons increase the escape flux, and thus the electrostatic potential difference that warrants the equality of the proton and electron fluxes. So, the presence of suprathermal electrons increases the electric force and accelerates the wind to high bulk velocities (Pierrard and Lemaire, 1996; Maksimovic et al., 1997a). High bulk velocities as observed in the high speed SW can be obtained in these models with a realistic coronal temperature simply by assuming electron VDFs with enhanced suprathermal tails, as indeed observed in situ in the SW.

Plasma wave turbulence is directly observed in solar wind and terestrial plasmas, and wave-particle interactions may therefore influence the propagation and acceleration of electrons and ions. In order to study the evolution of the electron distribution function in the presence of a spectrum of transverse waves fully kinetic dispersion models have been developed (Schlickeiser, 1989; Dröge et al., 1993; Steinacker and Miller, 1992; Schlickeiser, 1999; Vainio, 2000; Schlickeiser et al., 2010). Assuming transverse waves with a power-law spectral density, the pitch-angle diffusion mean-free-path $(\mathrm{mfp})$ and the acceleration time scales have been calculated for different energies of the electrons and ions (Steinacker and Miller, 1992; Schlickeiser, 1999; Vainio, 2000; Schlickeiser et al., 2010).

The acceleration of electrons by whistlers has been proposed as a first justification for the formation of generalized Lorentzian (or Kappa) particle distribution functions in interplanetary plasmas (Ma and Summers, 1999). Whistlers were assumed to be generated below the solar coronal base and transmited through the corona into interplanetary space. The simulations have shown that even in a quiet solar corona, the resonant interaction with whistlers are capable of generating suprathermal electrons and enhanced fluxes in the SW reproducing the observed features of the VDFs components, i.e., core, halo, strahl and superhalo components (Vocks and Mann, 2003; Vocks et al., 2008).

The role of whistler waves has been extended to large radial distances in the SW based on the observed magnetic field fluctuations. Further presence of the whistler waves at large radial distances in the SW can influence the electron 
VDFs and explain the formation of both the halo and strahl components and a more isotropic VDF at higher energies (Vocks et al., 2005). Simplified diffusion models of scattering of the solar electrons due to resonant interaction with whistlers in interplanetary space cannot however explain the electron arrival delays ( 10 mins) observed at 1 AU (Vocks and Mann, 2009). It was therefore suggested that including both effects of the Coulomb collisions and the resonant wave scattering could probably resolve such disagreements.

The exospheric (collisionless) models mentioned above have the advantage to be analytic and to reproduce the basic mechanisms of the SW acceleration. Nevertheless, such models cannot reproduce all the SW characteristics: they lead to excessively large temperature anisotropies and unrealistic truncated VDFs. With the inclusion of Coulomb collisions a significant improvement has been made reproducing more constrained temperature anisotropies of the electron VDF in the SW.

In the present work, we analyze the effects of the resonant wave-particle (subscript " $w p ")$ interaction in addition to the Coulomb collisions (subscript " $c c "$ ). The electrons are considered in uniform fields and a superposed turbulent whistler wave spectrum. Only the slab modes propagating parallel to the interplanetary magnetic field are invoked because the energy exchange with oblique waves is expected to be less significant. The quasi-linear wave-particle scattering is described by the Fokker-Planck equation (FPE), a model (Schlickeiser, 1989; Vainio, 2000) successfully applied to electron acceleration in solar flares (Steinacker and Miller, 1992) and SW (Dröge et al., 1993). A similar method of solution is used as in Pierrard et al. (1999) and Pierrard et al. (2001), based on spectral expansion of the VDF in orthogonal polynomials. The same approach can also be used to test the effects of other types of interactions (Pierrard, 2010). The effects of kinetic Alfven waves on the formation of the proton beams have been analyzed by Pierrard and Voitenko (2010).

\section{Description of the Model}

The kinetic transport equation for the evolution of the velocity distribution function $f(\mathbf{r}, \mathbf{v}, t)$ of the electrons in the $\mathrm{SW}$ is:

$$
\frac{\partial f(\mathbf{r}, \mathbf{v}, t)}{\partial t}+\left(\mathbf{v} \cdot \nabla_{\mathbf{r}}\right) f(\mathbf{r}, \mathbf{v}, t)+\left(\mathbf{a} \cdot \nabla_{\mathbf{v}}\right) f(\mathbf{r}, \mathbf{v}, t)=\left(\frac{d f}{d t}\right)_{c c}+\left(\frac{d f}{d t}\right)_{w p}
$$

where $\mathbf{r}$ and $\mathbf{v}$ are respectively the position and velocity vectors of the particles, $\mathbf{a}$ is the acceleration due to external forces and $t$ is the time. We are interested by the steady state solution of this equation. In the case of the SW, the forces are the electric force $Z e \mathbf{E}$, the gravitational force $m \mathbf{g}$ and the Lorentz force resulting from the magnetic field distribution. The acceleration for the electrons is thus given by:

$$
\mathbf{a}=\left(\frac{-e \mathbf{E}}{m}+\mathbf{g}\right)-\frac{e}{m}(\mathbf{v} \times \mathbf{B})=\mathbf{a}_{\mathbf{r}}(\mathbf{r})+\mathbf{a}_{\mathbf{L}}
$$


where $e$ is the electric charge of electrons, $m$ their mass, $\mathbf{E}$ the electric field, $\mathbf{g}$ the gravitational acceleration, $\mathbf{B}$ the magnetic field (assumed to decrease as $\left.r^{-2}\right), \mathbf{a}_{\mathbf{r}}(\mathbf{r})$ is the radial component of the acceleration due to the electric and gravitational forces that are vertical and $\mathbf{a}_{\mathbf{L}}$ is the non radial term due to the Lorentz force.

In the coordinates of the radial distance $r$, the velocity $v$ and $\mu=\cos \theta=\hat{\mathbf{B}}_{0} \cdot \hat{\mathbf{k}}$ where $\theta$ is the pitch angle between the velocity vector and the magnetic field direction (assumed to be radial), the left hand side of eq.(1) becomes:

$$
D f=\frac{\partial f}{\partial t}+v \mu \frac{\partial f}{\partial r}+a_{r}(r)\left(\mu \frac{\partial f}{\partial v}+\frac{\left(1-\mu^{2}\right)}{v} \frac{\partial f}{\partial \mu}\right)+\frac{v}{r}\left(1-\mu^{2}\right) \frac{\partial f}{\partial \mu}
$$

(see Pierrard, 1997, for more details).

The term $(d f / d t)_{c c}$ on the right hand side of equation (1) represents the effects of the Coulomb collisions, as considered in the previous work (Pierrard et al., 2001). Here we adopt in addition the wave-particle scattering term $(d f / d t)_{w p}$ described in the next section.

\section{Description of the Wave-Particle Interaction Term}

We evaluate the electron distribution functions from the corona to the SW by considering wave-particle resonant interactions. The diffusion approximation to the interplanetary transport of the solar energetic particles should give reliable results if the particle's scattering mean free path is small compared to the scales of the physical system.

If we compare the scattering frequency, $\nu \equiv 2\left(1-\mu^{2}\right)^{-1} D_{\mu \mu}$ or the mean free path (mfp) evaluated at $\sim 1 \mathrm{AU}$ (see the Appendix) for the wave-particle interaction with that of binary (Coulomb) collisions we find $\lambda_{w p} / \mathrm{AU}=0.01-0.2$ and $\lambda_{c c} / \mathrm{AU} \simeq 1$, that makes us clear that binary collisions are very rare, and the wave particle interactions can be determinant for the particle transport process in the SW.

We consider the cyclotron resonance of electrons with parallel transverse cold plasma waves: slab geometry of parallel propagating waves with respect to the guiding magnetic field $\mathbf{B}_{0}$. The resonant particles must satisfy

$$
\omega_{r}(k)=v \mu k_{\|}+n \Omega_{e}
$$

where $\omega_{r}$ and $k$ are the oscillatory wave-frequency and the wave-number, respectively, and $\Omega_{e}=|e| B_{0} /(m c)$ is the electron gyrofrequency. The integer $n \neq 0$ must be finite, because, for parallel waves only the cyclotron resonance occurs (the transit-time damping is not possible).

In the FPE the term that describes the evolution of the electron distribution function in the presence of wave turbulence is chosen of the form (Schlickeiser, 1989)

$$
\left(\frac{\partial f}{\partial t}\right)_{w p}=\frac{\partial}{\partial \mu}\left(D_{\mu \mu} \frac{\partial f}{\partial \mu}+D_{\mu p} \frac{\partial f}{\partial p}\right)
$$




$$
+\frac{1}{p^{2}} \frac{\partial}{\partial p} p^{2}\left(D_{p \mu} \frac{\partial f}{\partial \mu}+D_{p p} \frac{\partial f}{\partial p}\right) .
$$

where $p$ is the particle's momentum, $D_{\mu p}=D_{p \mu}$, and for nonrelativistic electrons (Steinacker and Miller, 1992)

$$
\begin{gathered}
\frac{D_{p p}}{\Omega_{e}\left(m_{e} c\right)^{2}}=\frac{\pi}{3} \frac{A}{a}\left(\frac{\beta|\mu|}{a}\right)^{\frac{s-1}{3}}\left(1-\mu^{2}\right) \\
\frac{D_{\mu p}}{\Omega_{e}\left(m_{e} c\right)}=-\frac{\pi}{3} \frac{A}{a}\left[\frac{\mu}{|\mu|}\left(\frac{\beta|\mu|}{a}\right)^{\frac{s-2}{3}}+\frac{\mu}{\beta}\left(\frac{\beta|\mu|}{a}\right)^{\frac{s-1}{3}}\right]\left(1-\mu^{2}\right) \\
\frac{D_{\mu \mu}}{\Omega_{e}}=\frac{\pi}{3} \frac{A}{a}\left[\left(\frac{\beta|\mu|}{a}\right)^{\frac{s-3}{3}}+\frac{2 \mu}{|\mu|} \frac{\mu}{\beta}\left(\frac{\beta|\mu|}{a}\right)^{\frac{s-2}{3}}\right. \\
\left.+\left(\frac{\mu}{\beta}\right)^{2}\left(\frac{\beta|\mu|}{a}\right)^{\frac{s-1}{3}}\right]\left(1-\mu^{2}\right)
\end{gathered}
$$

Here we have used $\beta=v / c, a=\omega_{p, e}^{2} / \Omega_{e}^{2}$, and assumed electrons resonantly interacting with right-handed polarized waves in the whistler regime and with a magnetic spectral density given by the power-law (Steinacker and Miller, 1992)

$$
\frac{|\delta B|^{2}}{B_{0}^{2}}=A \frac{c}{\Omega_{e}}\left|\frac{k c}{\Omega_{e}}\right|^{-s}, \text { for } \omega_{\min }<\omega_{r}<\Omega_{e} .
$$

The normalization constant $A=0.1$, and the spectral index is usually found in the interval $s=\frac{3}{2}-\frac{5}{3}$ and is expected to not exceed $s=2$ (Dröge and Schlickeiser, 1986; Steinacker and Miller, 1992).

\section{Spectral Method of Solution}

To obtain steady state solutions for the test electron VDF, we use a specialized spectral method similar to that described in Pierrard and Lemaire (1998) for the resolution of the FPE.

The dimensionless velocity is defined by

$$
x=\sqrt{\frac{m_{e}}{2 k_{B} T_{e}(r)}} v=\frac{v}{w_{e}(r)},
$$

where $w_{e}$ is the electron thermal speed and $k_{B}$ is the Boltzmann constant.

The solution is expanded in terms of orthogonal polynomials:

$$
\begin{aligned}
f(z, x, \mu)= & \exp \left(-x^{2}\right) \times \\
& \left(\sum_{l=0}^{n-1} \sum_{s=0}^{N-1} \sum_{m=0}^{M-1} a_{l s m} P_{l}(\mu) S_{s}(x) L_{m}(z)\right) .
\end{aligned}
$$


where $n, N$, and $M$ are integers whose value is ajusted to obtain the required numerical precision for the solution. We use Legendre polynomials $P_{l}(\mu)$ with respect to $\mu=\cos \theta$, speed polynomials $S_{s}(x)$ with respect to the normalized velocity $x$ and displaced Legendre polynomials on the interval $[0, c]$ with respect to the dimensionless altitude $z$. Note that Lorentzian polynomials have recently been developed (Magnus and Pierrard, 2008) and could be used as well to adjust the electron VDF since it can account the observed VDF tails decreasing as a power law of the square velocity instead of exponentially.

We use several successive steps in radial distance in view to have a finer scale in the corona where the density gradient is larger than at large distances. The $a_{l s m}$ coefficients are the coefficients of the expansion to be determined.

The boundary condition(s) determine the value of some coefficients $a_{l s m}$ at the refence level(s). The equation is solved numerically to find the $\operatorname{VDF} f(z, x, \mu)$ at the other altitudes. We choose 10 polynomials for each variable so that the results have a precision better than $10 \%$ with reasonably short CPU times. We have checked that the results do not change significantly when $n, N$ or $M$ is increased.

The advantage of this method is that in a discrete coordinate basis, the derivatives of any continuous function $f(y)$ can be approximated by the following expansion:

$$
\left(\frac{\partial f}{\partial y}\right)_{y=y_{i}} \simeq \sum_{j=0}^{N-1} D_{i j} f\left(y_{j}\right)
$$

where $D_{i j}$ are the matrix elements of the derivative operator in the polynomial basis.

The integrals to calculate the moments of the test electrons are also easily performed by numerical quadrature taking into account the polynomial expansion (Pierrard, 1997). The moments are calculated from the VDF found by solving the FPE. The density is given by:

$$
n(r)=\int f(r, v, \mu) d \mathbf{v}
$$

The bulk velocity is calculated by:

$$
\mathbf{u}(r)=\left(\int f(r, v, \mu) \mathbf{v} d \mathbf{v}\right) / n .
$$

The temperature corresponds to a second order moment of the VDF:

$$
T(r)=\frac{1}{3}\left(T_{\|}(r)+2 T_{\perp}(r)\right)
$$

where

$$
\begin{aligned}
T_{\|}(r) & =\frac{m \int\left(v_{\|}-u\right)^{2} f(r, v, \mu) d \mathbf{v}}{k_{B} n(r)} \\
T_{\perp}(r) & =\frac{m \int v_{\perp}^{2} f(r, v, \mu) d \mathbf{v}}{2 k_{B} n(r)} .
\end{aligned}
$$




\section{Background Electrons}

The test population of the SW electrons are submitted to Coulomb collisions and turbulence. To determine the effects of the Coulomb collisions, we need to start with a background electron population for which the density and temperature profiles are known. We determine them from the Lorentzian exospheric model developed by Lamy et al. (2003). In this exospheric model, the interaction terms $(d f / d t)_{c c}$ and $(d f / d t)_{w p}$ are neglected in equation (1), which then becomes the Vlasov equation. This model gives also the electrostatic potential profile that is so important in the acceleration term. Indeed, such models reproduce the SW acceleration due to the ambipolar electrostatic potential imposed by the equality between the proton and electron fluxes. This model allows us to start from low radial distances in the corona. Figure 1 shows the moments and the electrostatic potential difference obtained for the background electrons with this purely exospheric model from 2 to $14 \mathrm{Rs}$ using $\kappa=4.5, r_{0}=1.4 \mathrm{Rs}, n_{0}=10^{4}$ $\mathrm{cm}^{-3}$ and $T_{e}=8 \times 10^{5} \mathrm{~K}, T_{p}=10^{6} \mathrm{~K}$. With a smaller value of $\kappa$ (thus larger suprathermal tails) and/or a higher electron temperature, the SW bulk velocity would be higher.

\section{Results}

\subsection{Model with Two Boundary Conditions}

Plasma wave turbulence is inferred to exist near the solar surface and in the solar corona (Roberts, 1989). We want to test with this first simulation whether the turbulence could explain the enhanced population of suprathermal particle observed in the solar wind at larger radial distances. To determine the VDF of the particles in the transition region between the collision dominated plasma at low radial distance and the collisionless plasma, we consider boundary conditions both at low altitude and at high altitude. Similar collisional models of polar and solar wind with two boundary conditions have been developed by Barghouthi et al. (2001) based on particle in cell simulations and Lie-Svendsen et al. (1997) based on the resolution of the FPE by a finite difference numerical scheme.

At the bottom boundary $r_{0}=1.4 \mathrm{R}_{s}$, the outward moving particles are assumed to be distributed according to an isotropic and Maxwellian VDF:

$$
f\left(y, r_{0}, \mu>0\right)=n_{0}\left(\frac{m}{2 k T \pi}\right)^{3 / 2} \exp \left(-y^{2}\right)
$$

At the upper boundary $r_{1}=14 \mathrm{R}_{s}$, the VDF is assumed to be truncated like in exospheric models: there are no particles coming from the interplanetary space in the downward loss cone, except trapped and ballistic particles which have been reflected at higher altitudes because they had not enough energy to escape:

$$
\begin{aligned}
& f\left(y>y_{\mathrm{esc}}, r_{1}, \mu<0\right)=0 \\
& f\left(y<y_{\mathrm{esc}}, r_{1}, \mu<0\right)=f\left(y<y_{\mathrm{esc}}, r_{1}, \mu>0\right) .
\end{aligned}
$$



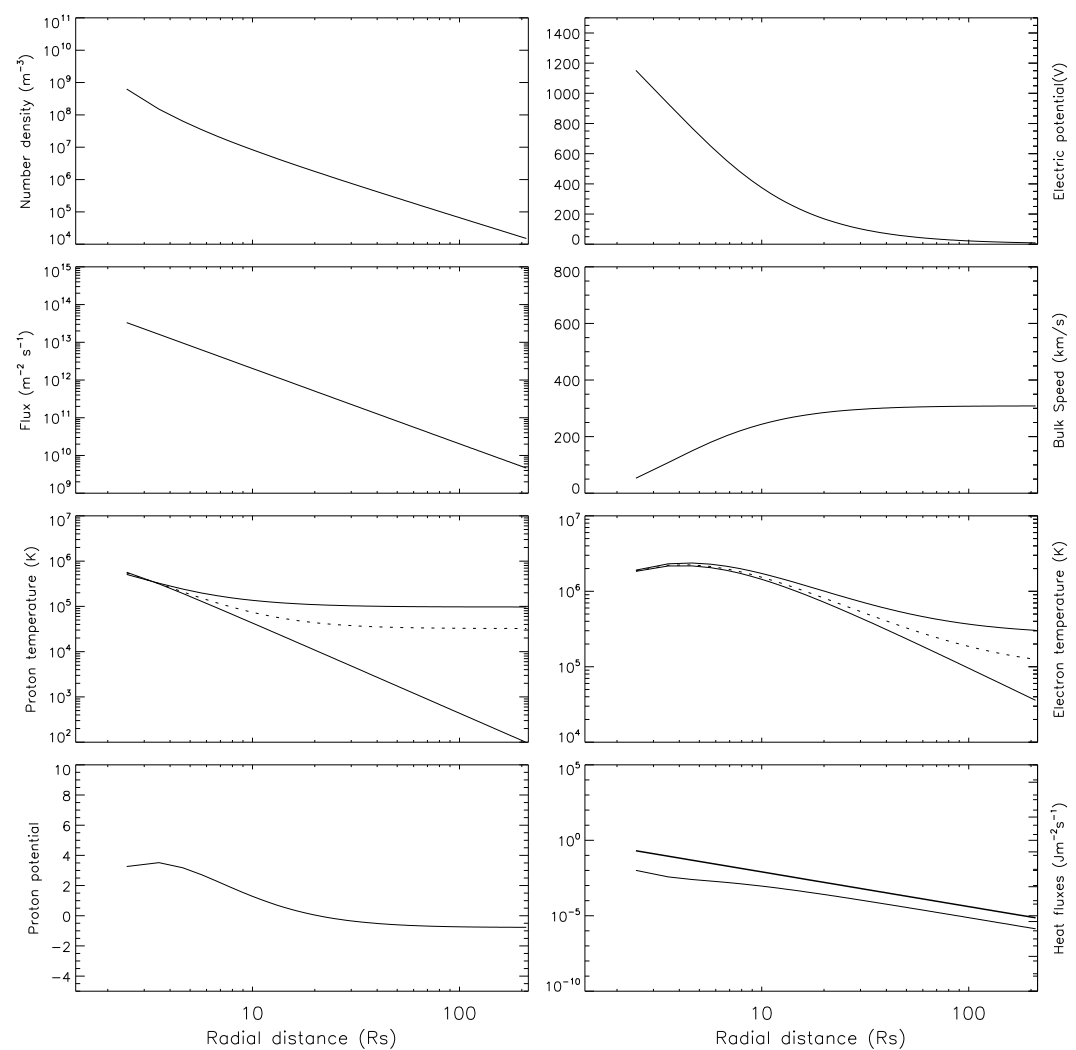

Figure 1. Profiles of the moments obtained with a purely exospheric model. The density, temperature and electric potential are used for the background particles. For the temperatures (panels 5 and 6 ), the upper line corresponds to the parallel temperature, the bottom line to the perpendicular temperature and the dashed line to the average temperature. For the heat flux (panel 8), the upper line corresponds to the electrons and the bottom line to the protons.

This choice of boundary conditions is the standard one and has been justified theoretically by arguing that collisions at low altitude maintain a nearly isotropic and Maxwellian VDF while at high altitude, the particles with energies high enough to escape never return back. The observations show that the VDF has not necessarily a Maxwellian (exponential) tail. In addition, the observed VDF is generally not so sharply truncated as imposed by (19). This assumption is based on the exospheric hypothesis and permits to provide the upward flux of particles and energy.

Figure 2 shows the VDF at 4 different altitudes and illustrates the anisotropy developed due to the boundary conditions. The tails of the VDF are also clearly modified, but more due to the truncation of the boundary conditions than due to the wave turbulence. Indeed, at these low radial distances, the waveparticle interaction mfp is large or comparable to the Coulomb collision mfp, 


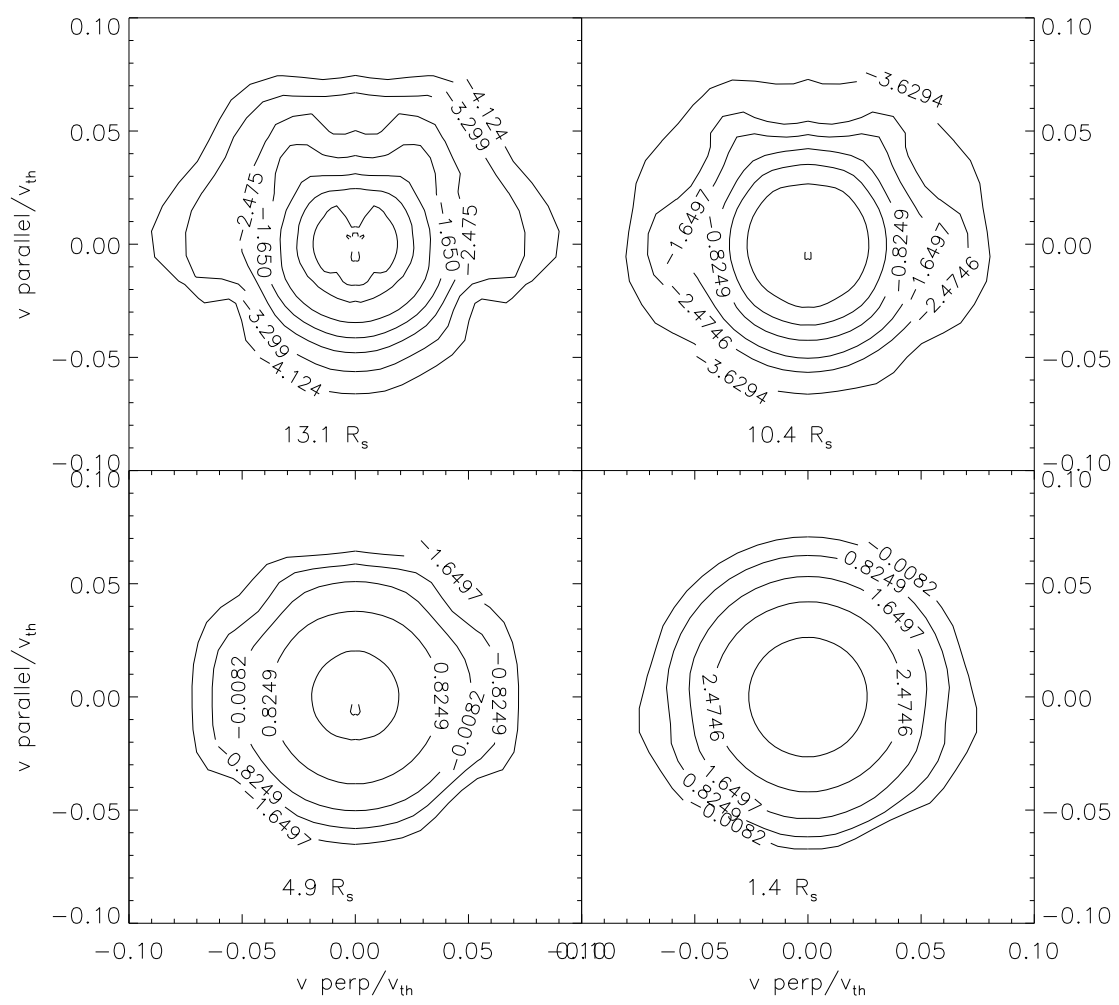

Figure 2. VDF obtained at different radial distances assuming 2 boundary conditions at 1.4 Rs and $14 \mathrm{Rs}$ by solving the FPE including whistler turbulence.

as illustrated in the Appendix. The wave turbulence term can only have effects in the regions where it becomes larger than the Coulomb collision term. The main moments, number density, bulk velocity and temperature profiles obtained within this model are displayed in Figure 3.

\subsection{Starting from Observations at $1 \mathrm{AU}$}

At $1 \mathrm{AU}$, the mfp of the wave-particle interaction is smaller or much smaller than that of the binary collisions showing that the effect of wave-turbulence should be considered in the evolution equation at large radial distances. In Figure 8 from Appendix, one can see that the turbulence mfp becomes comparable or less than the Coulomb mfp above few tens of Rs for standard boundary conditions.

The actual VDF at low altitude is unknown. To calculate it a-posteriori by solving the FPE, we choose now an alternative reasoning that lead us to fix the boundary conditions at 1 AU from WIND observations in order to determine the shape of the electron distributions at lower radial distances. 

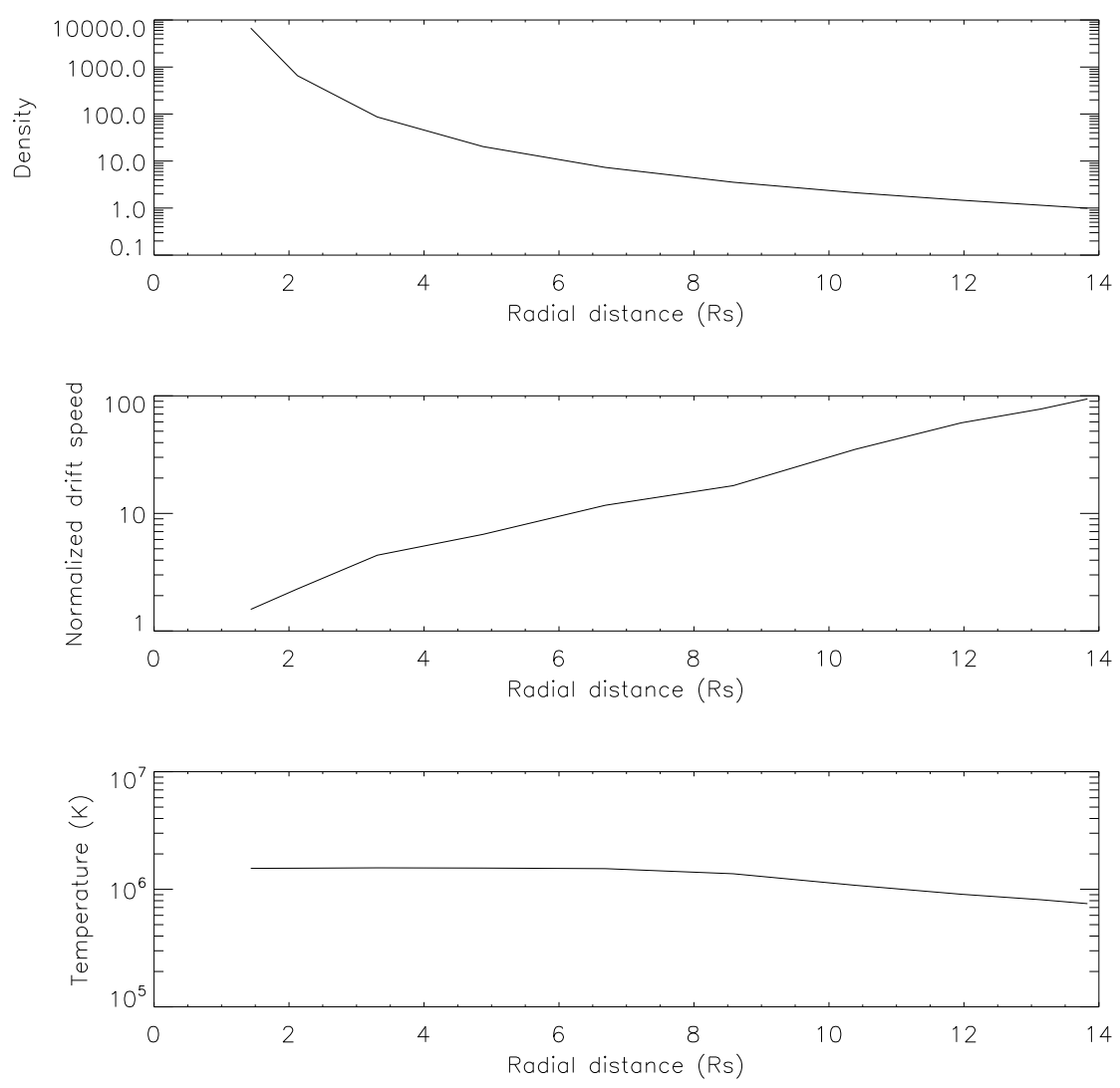

Figure 3. Number density, bulk velocity and temperature profiles obtained assuming 2 boundary conditions between 1.4 and 14 Rs by solving the FPE including whistler turbulence.

A typical electron VDF observed at $1 \mathrm{AU}$ in the low speed SW by the instrument 3DP aboard of WIND is illustrated on Figure 4. It is clearly characterized by large suprathermal tails in the parallel and perpendicular directions. These suprathermal tails are even larger in the high speed SW (Maksimovic et al., 1997b) but are also always observed in the low speed SW. The observed VDF is used as boundary condition to determine the electron VDF at lower radial distances. The results are presented in Figure 5 illustrating the VDF at different radial distances. Figure 6 shows the main moments: number density, bulk velocity, and temperature profiles. Again, the average values of these moments are not very affected by wave turbulence, contrary to the temperature anisotropy.

At lower radial distances, the VDF becomes anisotropic (asymmetric contours in Figure 5) with perpendicular velocity larger than the parallel one, while the boundary VDF had on the contrary a slight strahl component (the VDF was slightly aligned to the direction parallel to the magnetic field). This shows that the turbulence term affects the isotropy of the electron VDF and especially that 


\section{Velocity distribution function}

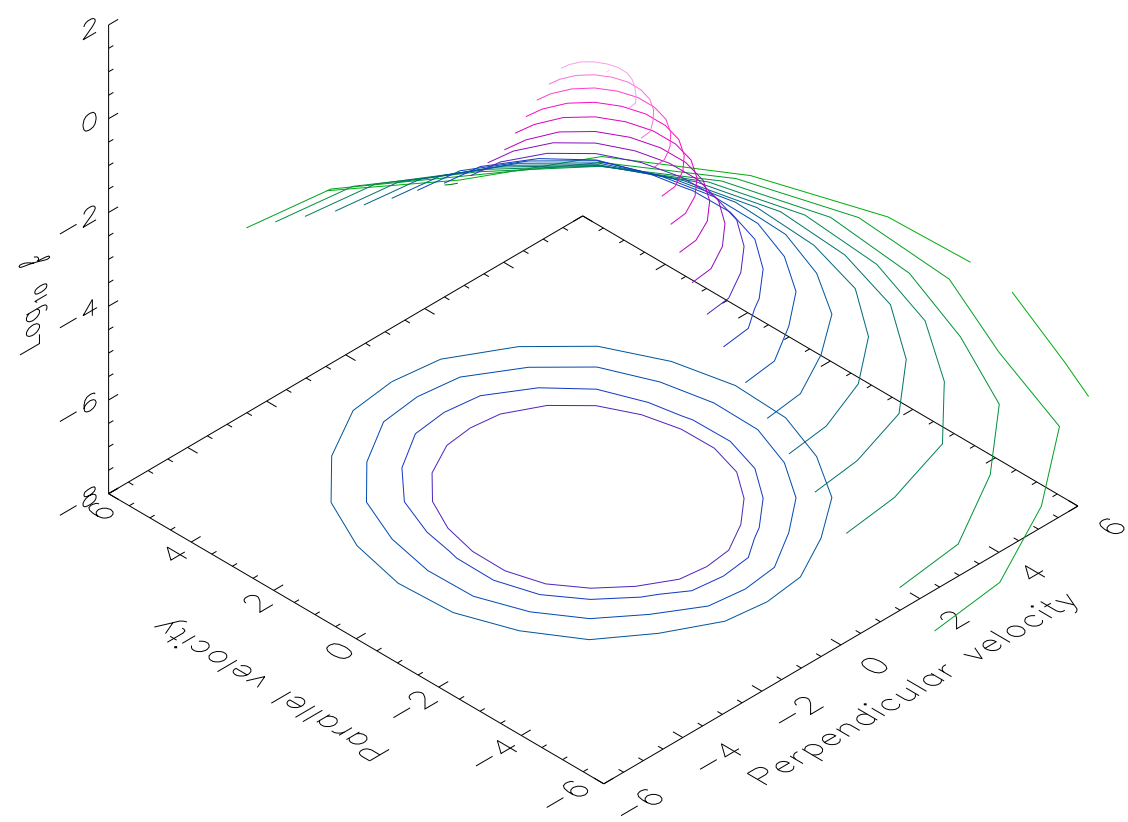

Figure 4. 3D display of the electron VDF observed at 1 AU by WIND.

the magnetic momentum of the electrons is not conserved, contrary to what is assumed in a purely exospheric model. This explains also why the exospheric models lead to excesively low perpendicular temperatures.

\section{Discussion}

A purely exospheric model leads to highly anisotropic VDF because the mirror force and the conservation of the magnetic moment focuses the strahl electrons in a narrow beam in the direction of the magnetic field. This beam is much more narrow than what is observed in the solar wind. That is why Vocks et al. (2005) have hypothesized scattering by INWARD propagating whistlers, even if there is no observation of such a population of inward propagating whistlers nor much theoretical reason for their excitation. In the present work, we show that the presence of Coulomb collisions and turbulence scattering is sufficient to enhance the electron diffusion to form a quasi-isotropic halo component. The 


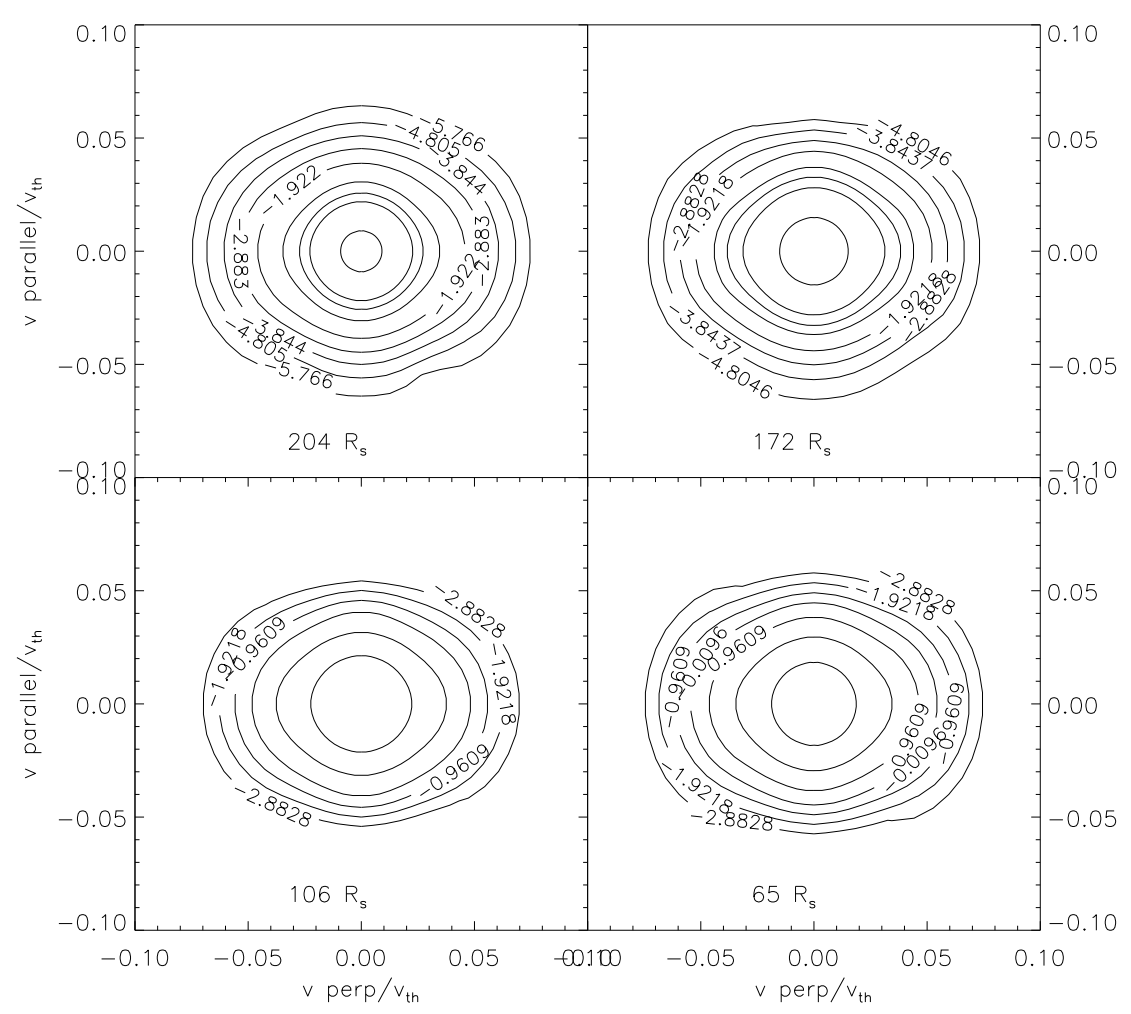

Figure 5. VDF obtained at different radial distances by solving the Fokker-Planck equation including wave-turbulence and using a VDF observed at $215 \mathrm{Rs} \simeq 1 \mathrm{AU}$.

strahl component with a finite width is due to the Lorentz force focusing the VDF in the magnetic field direction. The interactions between the particles modify mainly their directions and thus reduce the VDF anisotropy obtained in purely exospheric models without changing the average values of the lowest moments, in better agreement to the VDFs obtained at large radial distances in Figure 2. Moreover, the assumed boundary conditions play a very important role in the characteristics of the VDF obtained by solving the evolution equation.

Stverak et al. (2008) have shown that electron Coulomb collisions may have an effect of maintaining a low temperature anisotropy of the bulk population in the SW, while the large departures from isotropy are constrained by whistler and firehose instabilities. In turn, the energization of charged test particles was also studied by Shizgal (2007) who showed that the VDF tends to Maxwellian in presence of Coulomb collisions and in the absence of wave-particle interactions. When wave-particle interactions are included, an initial distribution tends to a steady state VDF with nonequilibrium (non-maxwellian) tails and associated to 

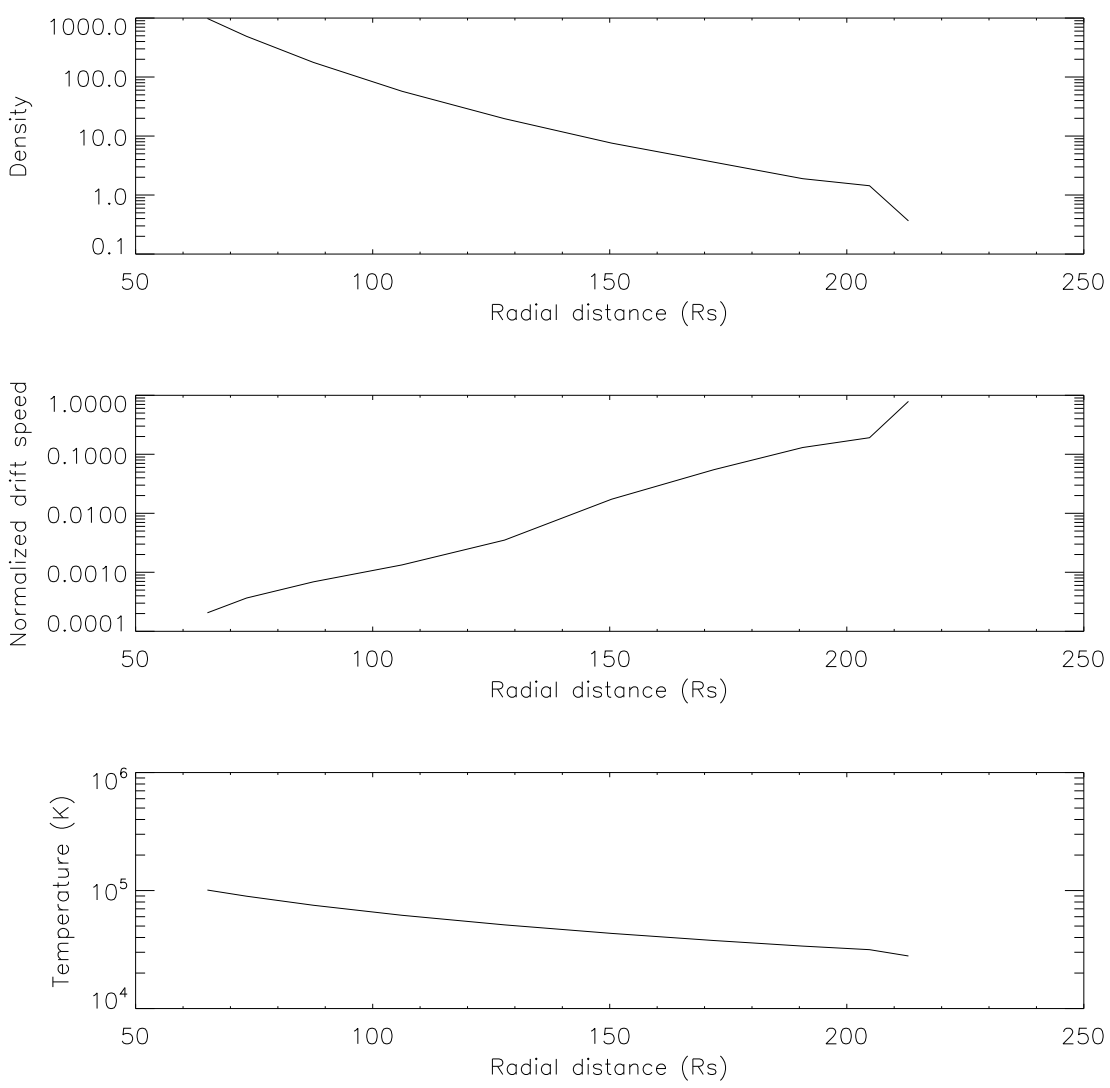

Figure 6. Number density, bulk velocity and temperature profiles obtained by solving the Fokker-Planck equation including wave-turbulence and using a VDF observed at 215 Rs $\simeq 1$ AU.

an increase of the entropy (Leubner, 2004). He considers only $D_{p p}$ which is the most important coefficient for treatment of acceleration. In our case, we consider also the components depending on $\mu$ and they seem to have more effects than $D_{p p}$ at large radial distances. Shizgal (2007) shows that the VDF tends to Kappa distributions if the diffusion coefficient is in $1 / v$. The choice of the wave-particle diffusion coefficient is crucial in the energization process.

The presence of the wave-turbulence term shows some differences for the VDF isotropy but do not modify the main moments. That is not so surprising because for electrons, the turbulence is limited to whistler waves. The bulk velocity is mainly determined by the electric force accelerating the solar particles outside. The acceleration term is dominant on the interaction terms, even when considering the wave turbulence.

The SW and even the coronal plasma are not dominated by collisions, since the mfp is already larger than the density scale height at very low radial distances 

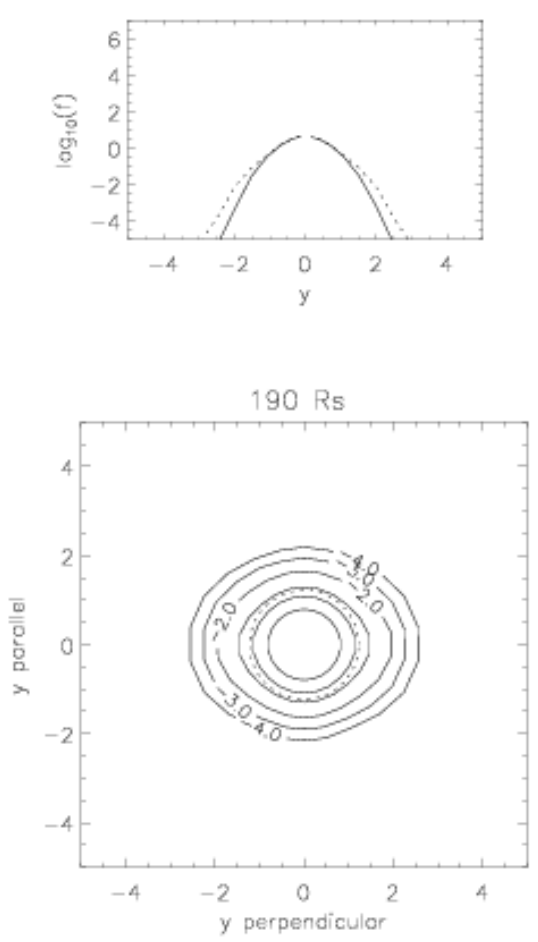

Figure 7. The electron VDF contours found at 190 Rs in the velocity space (bottom panel) and sections (top panel) in the direction parallel (solid line) and perpendicular (dashed line) to the magnetic field.

(Pierrard and Lamy, 2003; Meyer-Vernet, 2006). Even at lower radial distances, the plasma can not be considered to be dominated by collisions due to the long range properties of the Coulomb interaction. Indeed, since the particle mfp increases as $v^{4}$ in a plasma, the suprathermal particles are non collisional even when thermal particles are submitted to many collisions. In the solar transition region (from the corona to the wind), the heat flux is not classical (Shoub, 1983). The VDF of the particles is not well represented by a Maxwellian VDF in such regions where strong gradients of temperature and of density are observed. The presence of nonthermal electrons in the solar transition of active regions was evidenced observationally by SiIII line ratios from SUMER (Pinfield et al., 1999).

At $1 \mathrm{AU}$ the resonant interaction between electrons and whistler fluctuations are, in general, more efficient than Coulomb collisions. This feature can extend to smaller radial distances (few solar radii), where according to the radial mfp profiles derived in the Appendix (based on small values of the turbulent mfp at $\sim 1 \mathrm{AU}$, e.g., $\lambda_{w p, 0} \simeq 0.01 \mathrm{AU} \ll \lambda_{c c, 0} \simeq 1 \mathrm{AU}$ ), binary collisions may be dominated again by the resonant wave-particle interactions. This indicates that, even at low altitudes in the SW, the acceleration via resonant interaction 
with parallel electromagnetic waves should be taken into account as a plausible mechanism of energizing the electrons, and thus explaining the existence of nonthermal distributions in these regions. Here we have analyzed the pitch-angle diffusion as it is considered the basic effect of the whistler waves interaction on the electron VDF. The energization time scales, $\tau_{p p}=p^{2} / D_{p p}, \tau_{\mu p}=\left|p / D_{\mu p}\right|$, and $\tau_{\mu \mu}=1 / D_{\mu p}$ (and their radial profiles) can also be derived by using all three momentum diffusion coefficients (6), (7), and (8), respectively, but this will make the object of our next investigations.

\section{Conclusions}

We have investigated the influence of the whistler electromagnetic plasma turbulence on the evolution of the electron distribution function and the principal moments in the SW. In addition to the Fokker-Planck Coulomb collision term, an operator for turbulent wave-particle interaction has been added. Scattering of the solar electrons in the solar wind is caused by the interplay of Coulomb collisions and the resonant cyclotron interactions with slab whistler modes. At large radial distances contribution from the whistler turbulence is larger than that of the Coulomb collision, but the acceleration term from the guiding field dominates both of them. Non-Maxwellian distributions are observed in many space plasmas and for all particle species (electrons, but also protons and heavy ions). This is due to the low collision rate for Coulomb collisions compared to energization in rarefied space plasmas. Nonequilibrium steady state distributions are then obtained. The steady state solution of the Fokker-Planck Coulomb collision term is the Maxwellian VDF. At low radial distances, the density of the particles is so high that the mfp of the particle collisions is lower than the density scale height. So that it is expected that in the low corona, the VDF of the particles should be close to a Maxwellian, except if an additional mechanism can modify this VDF.

The results of our new approach are consistent with the observations and the complementary models. Adding a wave-turbulence term capable to energize the particles modifies the VDF, as shown by Shizgal (2007). The steady state solution is no longer Maxwellian in the regions where the wave-particle interaction term is larger than the Coulomb collision term. The wave-particle diffusion coefficients are then crucial. In the SW itself, the acceleration term is higher than the Coulomb and wave-particle interaction term. Wave-particle interaction can lead to VDFs that are non-Maxwellian, and the acceleration of electrons by whistlers was indeed the first justification for the formation of generalized Lorentzian (or Kappa) particle distribution functions in space plasmas (Ma and Summers, 1999). Vocks and Mann (2003) and Vocks et al. (2005) have also shown that the resonant interaction between electrons and the whistler waves generated below the solar coronal base can produce an enhancement of suprathermal electron flux compared to the core flux.

The acceleration term is dominant in the FPE. It is responsible of the SW acceleration. The wave turbulence do not modify the average values of the VDF. Compared to purely exospheric models, the solution of the FPE is more realistic, 
because the isocontours of the VDF are smooth and the temperature anisotropy is reduced. Even more than the interaction term, the results depend on the assumptions made at the boundary conditions.

We have calculated both radial profiles of the electron mfp for Coulomb collisions and for resonant interaction between electrons and the parallel whistler waves. Their comparison suggest, first, that wave fluctuations can be more efficient than Coulomb collisions even at very small radial distances. Second, the energization of electrons due to resonant interaction with parallel whistlers can be effective at very low altitudes, in regions where the acceleration was believed to be negligible due to Coulomb collisions. Thus, our present results extend the role of the wave turbulence to small altitudes in the outer corona where, if it is more efficient than the Coulomb collisions, it may then explain formation of suprathermal populations. This comes to confirm some indications that the electron VDF is non-Maxwellian in these regions.

\section{Appendix}

\section{A. Turbulence Scattering mfp and Collision mfp for the Solar Wind Electrons}

The quasilinear turbulence scattering mfp of the SW electrons is calculated from gyro-resonant interactions with a spectrum of parallel whistler modes with high frequencies in the interval $\Omega_{e}<\omega<-\Omega_{p}$, where $\Omega_{e, p}=e B /\left(m_{e, p} c\right)$ is the gyrofrequency for electrons (subscript $e$ ) or for protons (subscript $p$ ), $B$ is the mean interplanetary magnetic field, $m_{e}$ and $e$ are the mass and the elementary charge of particles (electron or proton) and $c$ is the speed of light in vacuum.

\section{A.1. Evaluation at $1 \mathrm{AU}$}

\section{A.1.1. Turbulence Scattering mfp for Electrons}

Using the simple formalism developed by Schlickeiser (1999) with some corrections introduced by Vainio (2000), the wave turbulence scattering mfp for electrons is found as

$$
\lambda_{w p} \simeq \frac{6}{\pi(s-1)\left(1-H_{c}^{2}\right)} \frac{c}{\omega_{p e}} \frac{B_{0}^{2}}{\delta B_{W}^{2}}\left(\frac{\Omega_{e}}{\omega_{m}}\right)^{s-1} \gamma^{2-s} \mu^{-1 / 2}
$$

where $\omega_{p e}=4 \pi n e^{2} / m_{e}$ is the plasma frequency, $\mu=m_{p} / m_{e}$ is the protonelectron mass ratio, $s$ is the spectral index of the power-law spectrum $(s=$ $5 / 3), H_{c}<1$ is the cross helicity, usually very small, $H_{c} \simeq 0, \omega_{m} \equiv \omega_{\min }$ is the minimum frequency observed, $\delta B_{W}$ is the small amplitude of the whistler magnetic field fluctuations (usually with a small normalized density $\delta B_{W}^{2} / B_{0}^{2} \simeq$ 0.01 ), and the relativistic effects are neglected taking a Lorentz factor $\gamma=1$.

In order to evaluate the mfp at $1 \mathrm{AU} \simeq 215 R_{s} \simeq 10^{12} \mathrm{~m}$, we use typical values observed at this altitude in the SW for the plasma, the regular magnetic field and the turbulent field (Schlickeiser, 1999; Vainio, 2000): the plasma skin depth 
$c / \omega_{p e, 0} \simeq 10^{-8} \mathrm{AU}\left(n_{e, 0} \simeq 10 \mathrm{~cm}^{-3}\right), \Omega_{e, 0} / \omega_{m}=1.8 \times 10^{7}, s=5 / 3, H_{c} \simeq 0$, and $\delta B_{W}^{2} / B_{0}^{2} \simeq 0.01$. With these values in (21) we find the turbulence scattering mfp for electrons at $1 \mathrm{AU}$ is $\lambda_{w p}(1 \mathrm{AU}) \equiv \lambda_{w p, 0} \simeq 0.01 \mathrm{AU}$. Only occasionally, variations of the density or the energy of electrons, or the whistler wave density could enhance the mfp with one order of magnitude, but the maximum values do not exceed $\lambda_{w p, 0}<0.2 \mathrm{AU}$.

\section{A.1.2. Collisional mfp for Electrons}

The mfp for close Coulomb collisions of electrons is given by

$$
\lambda_{c c} \simeq \frac{1}{\sigma n}=\frac{1}{\pi r_{e}^{2} n}=\frac{1}{\pi n}\left(\frac{k_{B} T_{e}}{e^{2}}\right)^{2}
$$

where $\sigma=\pi r_{e}^{2} \simeq 10^{-9} \mathrm{~m}^{2} \mathrm{~K}^{2} / T_{e}^{2}$ is the cross-section for close collisions, (at a distance where the Coulomb potential energy is approximately equal to the kinetic thermal energy, $\left.e^{2} / r_{e} \sim k_{B} T_{e}\right)$. At $1 \mathrm{AU}$, the SW density and temperature take the mean values $n_{e, 0} \simeq 10 \mathrm{~cm}^{-3}$ and $T_{e, 0} \simeq 1.5 \times 10^{5} \mathrm{~K}$, respectively, which lead in Eq. (22) to a collisional mfp $\lambda_{c c}(1 \mathrm{AU}) \equiv \lambda_{c c, 0} \simeq 1 \mathrm{AU}$. This is in agreement with the observations (Bieber et al., 1994; Dröge, 2003).

The mfp for collisions is much larger than the mfp for turbulence scattering, $\lambda_{c c, 0} \simeq 1 \mathrm{AU} \gg \lambda_{w p, 0} \simeq 0.01 \mathrm{AU}$, and the wave turbulence is expected to have an important contribution to the scattering of electrons in this region (1 AU) of interplanetary space. Large values of the cross helicity have to be adopted for a low-frequency turbulence, to make the values of the mfp for protons consistent with measurements (Vaino, 2000), but in the whistler regime there are poor observational records because the plasma instruments have much lower cadence than the magnetic field experiment. However, if we increase the cross correlation between velocity and magnetic field fluctuations the wave-particle mfp increases, e.g., for $H_{c}=0.7$, with a factor $\approx 2$, but this is too small to be significant in our comparative analysis.

\section{A.2. Radial Profiles}

The both mpfs for Coulomb collisions and the wave-particle interaction are expected to be variable with the altitude in the SW because plasma parameters (particle density and temperature) and the magnetic field change with the radial distance.

\section{A.2.1. Turbulence Scattering $m f p$}

In order to find the radial profile of the wave-particle mfp from (21) we take into account the radial expansion of the electron density and the solar magnetic field towards the Earth. From the Parker and exospheric models for the expansion of the solar wind the evolution law for the electron density is

$$
n(r)=n_{0} \frac{r_{E}^{2}}{r^{2}}
$$


also confirmed by the observations (Aschwanden, 2004), and where $r_{E}=1 \mathrm{AU}$, and $n_{0}=n\left(r_{E}\right) \simeq 10 \mathrm{~cm}^{-3}$. The plasma skin-depth increases as

$$
\frac{c}{\omega_{p e}(r)}=\frac{c}{\omega_{p e, 0}} \frac{r}{r_{E}}
$$

reaching the highest value at $r=r_{E}=1 \mathrm{AU}, c / \omega_{p e, 0} \simeq 2.2 \times 10^{-5} \mathrm{AU}$.

The wave-particle $\mathrm{mfp}$ refers to the direction of the dominating magnetic field component, which in the inner heliosphere close to the Sun is the radial component. According to the Maxwell laws (and confirmed by the observations (Aschwanden, 2004)) the radial component of the interplanetary magnetic field decreases after a law similar to (23). The radial evolution of the electron gyrofrequency will take the form

$$
\Omega_{e}(r)=\Omega_{e, 0} \frac{r_{E}^{2}}{r^{2}}
$$

where $B_{0}=B\left(r_{E}\right)=5 \times 10^{-9} \mathrm{~T}\left(\simeq 5 \times 10^{-5} \mathrm{G}\right)$, and $\Omega_{e, 0} / \omega_{m}=1.8 \times 10^{7}$. Substituting (24) and (25) in (21) we find the radial profile of the wave-particle interaction $\mathrm{mfp}$

$$
\lambda_{w p}(r)=\lambda_{w p, 0}\left(\frac{r_{E}}{r}\right)^{2 s-3} \simeq 0.01\left(\frac{r_{E}}{r}\right)^{1 / 3} \mathrm{AU},
$$

where $\lambda_{w p}\left(r_{E}\right) \equiv \lambda_{w p, 0} \simeq 0.01 \mathrm{AU}$, and $s=5 / 3$. The wave density is assumed constant and small $\delta B_{W}^{2} / B_{0}^{2} \simeq 0.01$.

\section{A.2.2. Collisional $m f p$}

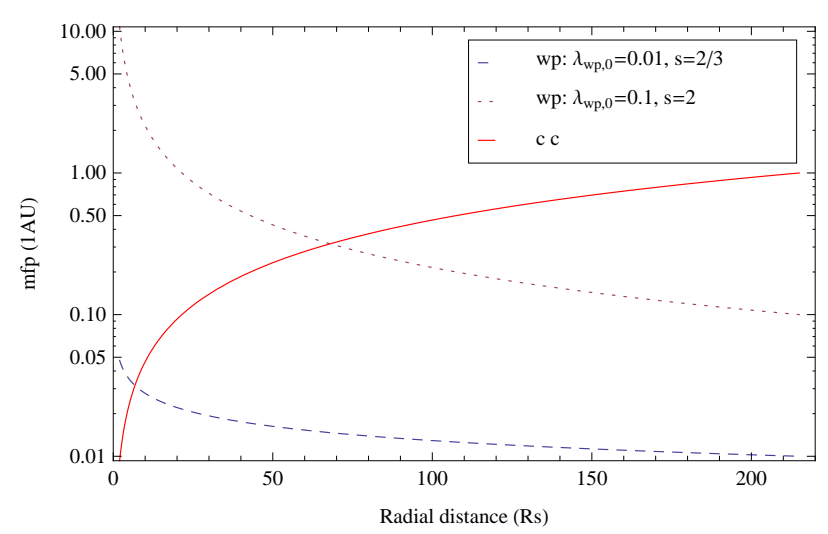

Figure 8. Radial profile of the mfp for the SW electrons: from Coulomb collisions (solid line) and wave-particle interaction (dashed and dotted lines) for two different boundary conditions at $1 \mathrm{AU} \simeq 215 \mathrm{Rs}$ (where $\mathrm{Rs}$ is the solar radius).

The collisional mfp (22) will have a radial evolution given by the radial law for density (23) and for the electron temperature (Richardson and Smith, 2003; 
Aschwanden, 2004)

$$
T_{e}(r)=T_{e, 0}\left(\frac{r_{E}}{r}\right)^{1 / 2}
$$

which also agrees with theoretical models (Pierrard et al., 1999). The electron temperature measured at $1 \mathrm{AU}$ is $T_{e}\left(r_{E}\right) \equiv T_{e, 0}=1.5 \times 10^{5} \mathrm{~K}$. Substituting (23) and (27) in equation (22) we find the collisional mfp

$$
\lambda_{c c}(r)=\lambda_{c c, 0} \frac{r}{r_{E}}=\frac{r}{r_{E}} \mathrm{AU}=r
$$

where $\lambda_{c c, 0} \simeq r_{E}=1$ AU.

Radial profiles of the mfps derived in (26) and (28) are shown in Figure 8. For the electron-whistler interactions the scattering $\mathrm{mfp}$ is displayed for two different boundary conditions, $\lambda_{w p, 0}=0.01 \mathrm{AU}$ and $s=5 / 3$ (dashed line) and $\lambda_{w p, 0}=$ $0.1 \mathrm{AU}$ and $s=2$ (dotted line). The observed mfp values are expected to not exceed the dotted line limit.

In coronal mass ejections detected at $1 \mathrm{AU}$ in the $\mathrm{SW}$ with small values of the electron mfp, e.g., $\lambda_{w p, 0} \simeq 0.01 \mathrm{AU}$, the wave-particle interactions could be more efficient than collisions even at very small radial distances, above a minimum critical altitude $r \geq r_{c} \simeq 6.5 \mathrm{Rs}$, given by the equality of the mfps (26) and (28), $\lambda_{w p}\left(r_{c}\right)=\lambda_{c c}\left(r_{c}\right) \simeq 0.003$ AU. The wave density was assumed constant and small $\delta B_{W}^{2} / B_{0}^{2} \simeq 0.01$, but this is expected to be increasing towards the Sun, reaching for instance, $\delta B_{W}^{2} / B_{0}^{2} \simeq 0.05$, or 0.1 , and making the mfp of the wave-particle interaction even lower in that region.

Acknowledgements V. Pierrard thanks the Solar Terrestrial Center of Excellence, the Belgian Institute for Space Aeronomy and BELSPO for their support. M. Lazar acknowledges support from the Katholieke Universiteit Leuven, Belgium and from the Research Department - Plasmas with Complex Interactions, Ruhr-Universität Bochum. R. Schlickeiser acknowledges support from the Deutsche Forschungsgemeinschaft through grants Schl 201/19-1 and Schl 201/21-1.

\section{References}

Aschwanden, M. J.: 2004, Physics of the Solar Corona, Springer, New York.

Barghouthi, I., Pierrard, V., Barakat, A.R., Lemaire, J.: 2001, Astrophys. Space Sci. 277, 427. Bieber, J.W., Matthaeus, W.H., Smith, C.W., Wanner, W., Kallenrode, M.-B., Wibberenz, G.: 1994, Astrophys. J. 420, 294.

Dröge, W.: 2003, Astrophys. J. 389, 1027.

Dröge, W., Schlickeiser, R.: 1986, Astrophys. J. 305, 909.

Dröge, W., Achatz, U., Wanner, W., Schlickeiser, R., Wibberenz G.: 1993, Astrophys. J. 407, L95.

Lamy, H., Pierrard, V., Maksimovic, M., Lemaire, J.: 2003, J. Geophys. Res. 108, 1047.

Lemaire, J., Pierrard, V.: 2001, Astrophys. Space Sci. 277, 2, 169.

Leubner, M.P.: 2004, Astrophys. J. 604, 469.

Lie-Svendsen, O., Hansteen, V.H., Leer, E.: 1997, J. Geophys. Res. 102, 4701.

Lie-Svendsen, O., Leer, E.: 2000, J. Geophys. Res. 105, 35.

Ma, C., Summers, D.: 1999, Geophys. Res. Lett. 26, 1121.

Magnus, A.P., Pierrard, V.: 2008, J. Comput. Appl. Math. 219, 431.

Maksimovic, M., Pierrard, V., Lemaire, J.: 1997, Astron. Astrophys. 324, 725. 
Maksimovic, M., Pierrard, V., Riley, P.: 1997, Geophys. Res. Let. 24, 9, 1151.

Meyer-Vernet, N.: 2006, Proceedings IAU Symposium 233, 269.

Pierrard, V.: 1997, Fonctions de distribution des vitesses des particules s'échappant de l'ionosphère, PhD thesis, UCL, Aeronomica Acta A 401.

Pierrard, V.: 2010, Astronum2009 Proceedings, ASP Conference series, Vol. 406.

Pierrard, V., Lamy, H.: 2003, Solar Physics 216, 47.

Pierrard, V., Lamy, H., Lemaire, J.: 2004, J. Geophys. Res. 109 A02118.

Pierrard, V., Lemaire, J.: 1996, J. Geophys. Res. 101, 7923.

Pierrard, V., Lemaire, J.: 1998, J. Geophys. Res. 103, 11701

Pierrard, V., Maksimovic, M., Lemaire, J.: 1999, J. Geophys. Res. 104, 17021.

Pierrard, V., Maksimovic, M., Lemaire, J.: 1001, J. Geophys. Res. 107, 29.305.

Pierrard, V., Voitenko, Y.: 2010, AIP Proc. of the 12th International Solar Wind Conference, 2009 1216, 102.

Pinfield, D.J., Keenan, F.P., Mathioudakis, M., Phillips, K.J.H., Curdt, W., Wilhelm, K.: 1999, Astrophys. J. 527, 1000.

Richardson, J.D., Smith, C.W.: 2003, Geophys. Res. Lett. 30, 1206.

Roberts, D.A.: 1999, J. Geophys. Res. 94, 6899.

Schlickeiser, R.: 1989, Astrophys. J. 336, 243.

Schlickeiser, R.: 1999, Astron. Astrophys. 351, 382

Schlickeiser, R., Lazar, M., Vukcevic, M.: 2010, Astrophys. J. in press

Shizgal, B.D.: 2007, Astrophys. Space Sci. 312, 227.

Shoub, E.C.: 1983, Astrophys. J. 266, 339.

Steinacker, J., Miller, J. A.: 1992, Astrophys. J. 393, 764.

Stverak, S., Travnicek, P., Maksimovic, M., Marsch, E., Fazakerley, A.N., Scime, E.E.: 2008, J. Geophys. Res. 113, A03103.

Vainio, R.: 2000, Astrophys. J. Suppl. Series 131, 519.

Vocks, C., Mann, G.: 2003, Astrophys. J. 593, 1134.

Vocks, C., Salem, C., Lin, R.P., Mann, G.: 2005, Astrophys. J. 627, 540.

Vocks, C., Mann, G., Rausche, G.: 2008, Astron. Astrophys. 480, 527.

Vocks, C., Mann, G.: 2009, Astron. Astrophys. 502, 325. 